University of Wollongong

Research Online

Faculty of Engineering - Papers (Archive)

Faculty of Engineering and Information

Sciences

2003

Observation of vortex distribution in samples of Bi-2223 Ag-sheathed tapes with and without uranium doping by means of the high-resolution Bitter method

S. Ohshima

Yamagata University, Japan

K. Ujiie

Yamagata University, Japan

T. Kawai

Yamagata University, Japan

K. Moriai

Yamagata University, Japan

H. Yamada

Yamagata University, Japan

See next page for additional authors

Follow this and additional works at: https://ro.uow.edu.au/engpapers

Part of the Engineering Commons

https://ro.uow.edu.au/engpapers/60

Recommended Citation

Ohshima, S.; Ujiie, K.; Kawai, T.; Moriai, K.; Yamada, H.; Marinaro, D. G.; and Dou, S. X.: Observation of vortex distribution in samples of $\mathrm{Bi}-2223 \mathrm{Ag}$-sheathed tapes with and without uranium doping by means of the high-resolution Bitter method 2003.

https://ro.uow.edu.au/engpapers/60

Research Online is the open access institutional repository for the University of Wollongong. For further information contact the UOW Library: research-pubs@uow.edu.au 


\section{Authors}

S. Ohshima, K. Ujiie, T. Kawai, K. Moriai, H. Yamada, D. G. Marinaro, and S. X. Dou 


\title{
Observation of Vortex Distribution in Samples of Bi-2223 Ag-Sheathed Tapes With and Without Uranium Doping by Means of the High-Resolution Bitter Method
}

\author{
S. Ohshima, K. Ujiie, T. Kawai, K. Moriai, H. Yamada, D. G. Marinaro, and S. X. Dou
}

\begin{abstract}
We examined the distribution of vortices in both Bi-2223 Ag-sheathed tape and U-doped Bi-2223 Ag-sheathed tape applying the high-resolution Bitter method. In the low temperature region $\left(T \ll T_{c}\right)$, we found that the vortices in the undoped Bi-2223 Ag-sheathed tape developed into either a triangular arrangement or a heterogeneous arrangement along the grain boundaries of the Ag sheath, which form weak pinning centers. The vortex distribution for $\mathrm{U}$-doped Bi-2223 samples was slightly different from the distribution for the undoped material. We observed some movement of the vortices in both the U-doped and the undoped Bi-2223 Ag-sheathed tapes at $50 \mathrm{~K}$. At higher temperatures, $T>75 \mathrm{~K}$, the vortices in the Bi-2223 tapes moved even faster so that no deposition pattern, indicating the location for vortices, formed.
\end{abstract}

Index Terms-Bi-2223, high-temperature superconductors, uranium doping, vortex.

\section{INTRODUCTION}

B i-2223 Ag-sheathed tape conductor is already applied practically in superconducting magnets that operate at liquid nitrogen temperatures. At temperatures of $77 \mathrm{~K}$, however, these magnets cannot generate high magnetic fields, since effective pinning centers do not exist. Therefore, a great deal of research has focused on introducing artificial pinning centers, e.g., by adding various compounds to Bi-2223 [1]-[3] and by changing the conditions of the cold rolling process and the heat treatment [4]-[6]. Recently, Marinaro et al. introduced point defects into Bi-2223 tapes, which improved $J_{c}$ in the high magnetic field region [7], [8]. They irradiated a U-doped Bi-2223 sample with thermal neutrons to artificially introduce point defects. The sample exhibited improved $J_{c}$ in a high magnetic field after the irradiation. It was proven that there

Manuscript received August 4, 2002. This work was supported in part by the Grant-in Aid from the Ministry of Education, Culture, Sports, Science and Technology of Japan.

S. Ohshima is with the Faculty of Engineering, Yamagata University, Yonezawa, 992-8510, Japan (e-mail: ohshima@yz.yamagata-u.ac.jp).

K. Ujiie, T. Kawai, K. Moriai, and H. Yamada are with the Faculty of Engineering, Yamagata University, Yonezawa, 992-8510, Japan.

D. G. Marinaro and S. X. Dou are with the Institute for Superconducting and Electronic Materials, University of Wollongong, Wollongong, NSW 2500, Australia.

Digital Object Identifier 10.1109/TASC.2003.812073 is an optimum level for both the U-doping and the degree of thermal neutron irradiation in order to improve $J_{c}$.

Direct observation of the vortices is a useful technique for evaluating the pinning centers and the pinning potential. We have succeeded in observing the distribution and movement of vortices in a Y-123 single crystal, a $\mathrm{Bi}-2212$ single crystal, a Y-123 QMG sample and in Bi-2223 Ag-sheathed tapes using the high-resolution Bitter method. The pinning potential and pinning centers were evaluated from the movement of the vortices. It was also clarified that the vortices moved with a hopping-like movement in the $\mathrm{Bi}-2223$ silver sheath tape at temperatures over $60 \mathrm{~K}$. This movement can be reduced if strong pinning sites exist in the sample. Therefore, it is interesting to examine the differences in the vortex distribution and movement in the samples with and without neutron irradiation.

\section{SAMPLE PREPARATION AND EXPERIMENTAL PROCEDURE}

The Bi-2223 Ag-sheathed mono-filamentary tapes were fabricated by the powder-in-tube method using a rolling process with an intermediate sintering step. The details of the fabrication process are reported elsewhere [9]. The U-doped Bi-2223 tapes were also fabricated by the powder-in-tube method. The powders that were used as precursors were doped with controlled amounts of ${ }^{235} \mathrm{UO}_{4}$ prior to processing. After processing, the samples were irradiated with highly moderated thermal neutrons in the HIFAR reactor at the Australian Nuclear Science and Technology Organization. The details of the fabrication process were reported in [8]. The specific combinations of ${ }^{235} \mathrm{UO}_{4}$ and the thermal neutron fluence are shown in Table I.

For the high-resolution Bitter method, we employed an evaporation technique using $\mathrm{Ni}$ wires. It is important to control the kinetic energy of the $\mathrm{Ni}$ particles in order to observe vortices in high-temperature superconductors. Therefore, the helium gas pressure in the chamber was accurately controlled in order to control the kinetic energy of the Ni particles. The samples were cooled in a magnetic field of $7.5 \times 10^{-3} \mathrm{~T}$, and then the $\mathrm{Ni}$ was deposited onto them. The final temperature, $T_{d}$, was varied between $25 \mathrm{~K}, 50 \mathrm{~K}$ and $75 \mathrm{~K}$. We examined the relationship between the distribution and movement of the vortices and temperature. The detailed observation process was reported in a previous paper [10], [11]. 
TABLE I

U-Doping Percentage and Total NeUtron IrRadiation FluEnCE

\begin{tabular}{|c|c|c|}
\hline \hline Sample & $\begin{array}{c}\text { Doping } \\
\text { percentage } \\
(\text { wt. } \%)\end{array}$ & $\begin{array}{c}\text { Thermal Neutron } \\
\text { Fluence } \\
\left(\times 10^{19} \mathrm{~m}^{-2}\right)\end{array}$ \\
\hline A & 0 & 0 \\
\hline B & 1.5 & 0.75 \\
\hline C & 1.5 & 3 \\
\hline
\end{tabular}

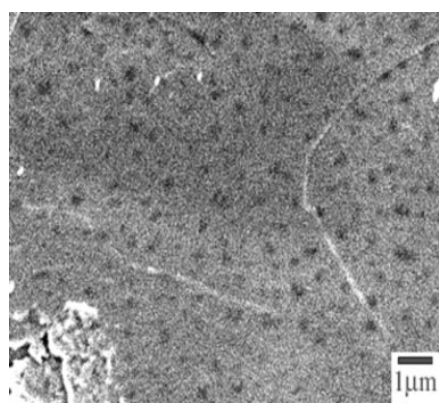

(a)

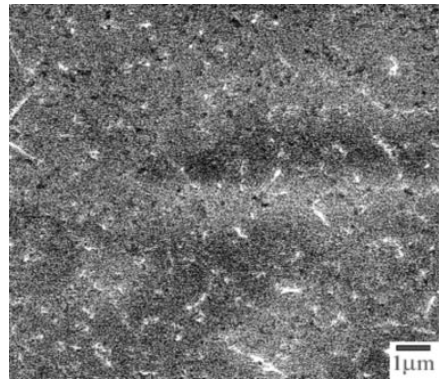

(c)

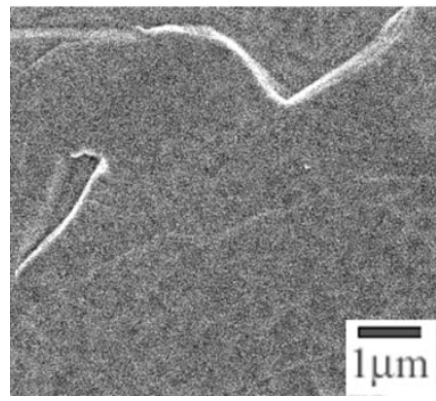

(e)

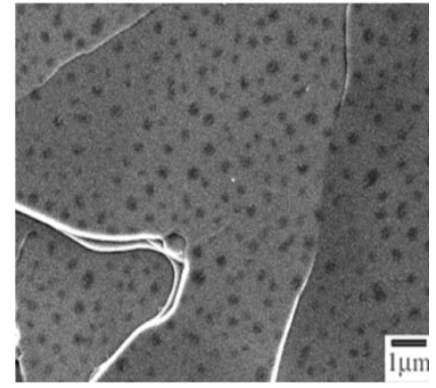

(b)

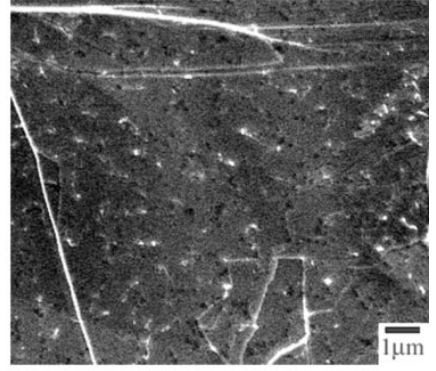

(d)

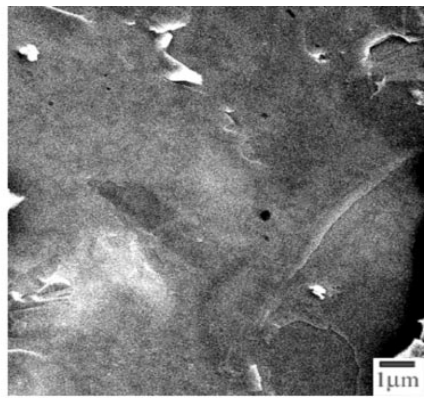

(f)
Fig. 1. (a) - (f). Vortex distribution of $\mathrm{Bi}-2223$ tapes with and without U-doping.(a), (c), (e): Sample A (without U-doping) (b), (d), (f): Sample $\mathrm{C}(1.5 \mathrm{wt} \%$ U-doping and irradiated with thermal neutrons at a fluence of $\left.3 \times 10^{19} \mathrm{~m}^{-2}\right)$. $\left(T_{d}\right.$ : The sample temperature while depositing nickel).

\section{RESULTS AND DISCUSSION}

\section{A. Effect of the Uranium Doping on the Vortex Distribution}

Fig. 1 shows the Ni decoration patterns for Bi-2223 tapes both with and without U-doping, as observed by SEM. Samples A and $\mathrm{C}$ are those shown in Table I. Ni was evaporated at $25 \mathrm{~K}$, $50 \mathrm{~K}$ and $75 \mathrm{~K}$, respectively. The temperature is shown as $T_{d}$ in the figure. The black dots in Fig. 1 show condensed Ni particles, i.e., they show the locations where the vortices existed. Therefore, we can explain the black dots (or lines) in the SEM pictures as being either a vortex or the location of a vortex. At
$25 \mathrm{~K}$, shown in Fig. 1(a) and (b), it was found that all of the locations of the vortices have circular shape. That is to say, the vortices did not move during the duration of the $\mathrm{Ni}$ deposition (30 seconds). Counting the black dots we saw that the density of the vortices in the uranium-doped sample (b) was slightly higher than that of the nondoped sample (a). The experiment was carried out by the magnetic-field-cooling method. Therefore, more vortices were trapped in the sample with the higher density of pinning centers.

It has been reported that dislocations linked to the nuclear fusion of the uranium can work effectively as pinning centers [7]. Therefore it was expected that, if sample $\mathrm{C}$ had a stronger pinning potential, the distribution of the vortices at higher temperature should change compared with that of sample A. As shown in Fig. 1(c) and (d), the shapes of the vortices changed from circular to slender spots. This means that the vortices moved due to their thermal energy while Ni was being deposited (30 seconds). It appeared that the vortices did not move randomly, but moved in a specific direction. This may be caused by a chain of dislocations formed by the neutron irradiation in the U-doped samples. The amount of movement was larger for the undoped sample (c). Fig. 1(e) and (f) show the Ni decoration pattern on the $\mathrm{Bi}-2223 \mathrm{Ag}$-sheathed tape, on which the Ni particles were deposited at $75 \mathrm{~K}$. As shown in this picture, the Ni particles did not precipitate into dots or lines. This means that the vortices moved quickly above $75 \mathrm{~K}$, so that the Ni precipitated into a cloudy pattern in both samples, A and C.

\section{B. Influence of the Thermal Neutron Fluence on the Vortex Distribution}

We examined the influence of the thermal neutron fluence on the vortex distribution of $\mathrm{Bi}-2223$ tapes with $1.5 \%$ uranium doping as shown for samples B and $\mathrm{C}$ in Table I. The neutron fluence for sample B was $0.75 \times 19^{19} \mathrm{~m}^{-2}$, and for sample $\mathrm{C}$ it was $3 \times 10^{19} \mathrm{~m}^{-2}$. Fig. 2(a) and (b) show the vortex distributions of samples $\mathrm{B}$ and $\mathrm{C}$. The sample temperature during $\mathrm{Ni}$ deposition was $25 \mathrm{~K}$. The vortices are shown as black dots. The shapes of the vortices were circular, indicating that all vortices were trapped in pinning centers at that temperature. We found that the vortices in Fig. 2(a) (sample B) arranged in a pattern of a triangular lattice. There was no regular pattern, however, in the distribution of the vortices of Fig. 2(b) (sample C). This difference may be caused by the difference in the pinning energy. In sample $\mathrm{C}$ irradiated at higher neutron fluence, stronger pinning existed, and the vortices became trapped in an irregular pattern. The vortices in sample B became formed a stable triangular lattice arrangement by the interaction between the vortices, since their pinning force was weaker.

Fig. 2(c) and (d) show the vortex patterns of samples B and C at $50 \mathrm{~K}$. The shapes of the vortices were not circular, but formed an elongated line indicating that the vortices moved during the $\mathrm{Ni}$ decoration process. The distribution patterns of samples B and $\mathrm{C}$ were slightly different. For sample B, shown in (c), the movement of the vortices was slightly larger than in sample $\mathrm{C}$ (d) indicating that the pinning potential of sample B was smaller than that of sample C. Fig. 2(e) and (f) shows the Ni decoration pattern of $\mathrm{Bi}-2223$ tapes at $75 \mathrm{~K}$. We did not observe any pattern in samples B and C. 


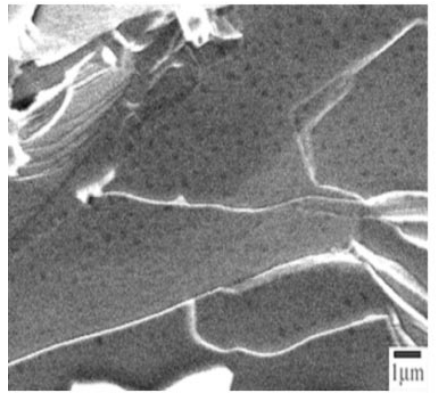

(a)

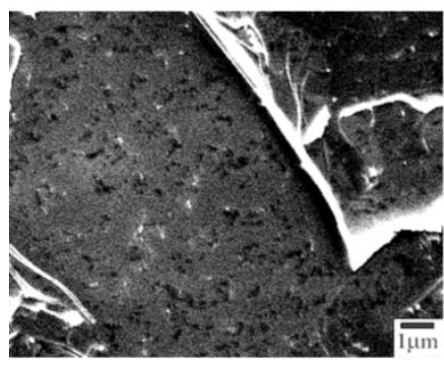

(c)

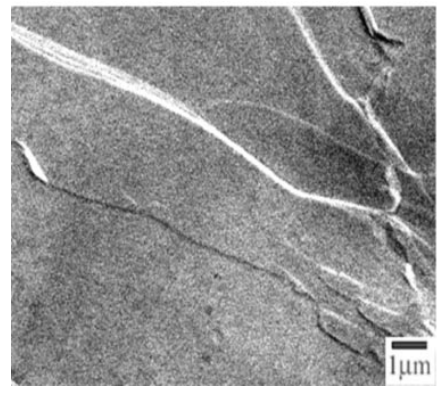

(e)

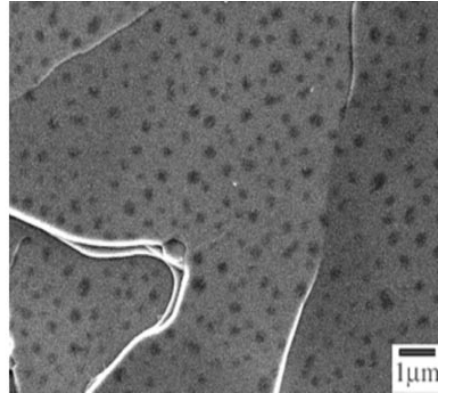

(b)

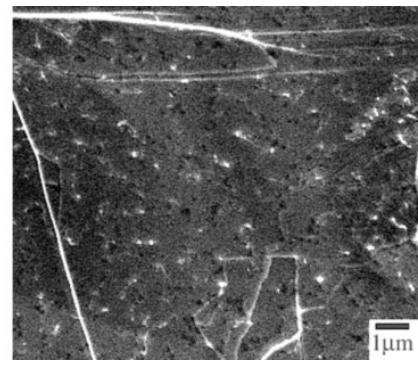

(d)

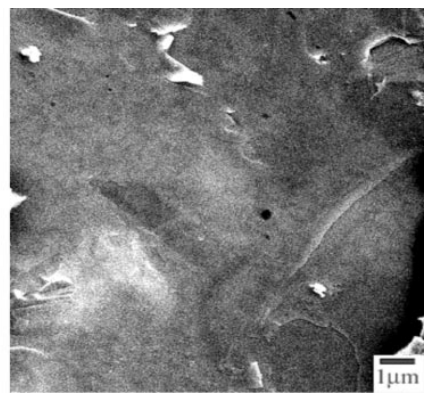

(f)
Fig. 2. (a) - (f). Vortex distribution of Bi-2223 tapes with U-doping varying the thermal neutron fluence.(a), (c), (e): Sample B (1.5 wt\% U-doping and irradiated with thermal neutrons at a fluence of $\left.0.75 \times 10^{19} \mathrm{~m}^{-2}\right)$. (b), (d), (f): Sample C (1.5 wt\% U-doping and irradiated with thermal neutrons at a fluence of $\left.3 \times 10^{19} \mathrm{~m}^{-2}\right)$.

Marinao et al. found that there is an improvement in flux pinning energy after neutron irradiation, and that an optimum doping and an optimum fluence level exists [7]. The normalized $J_{c}$ of the Bi-2223 Ag-sheathed tape with 2 wt. $\%{ }^{235} \mathrm{UO}_{3}$ doping measured at $0.8 \mathrm{~T}$ was about 250 times larger than that of the nonirradiated sample. We used the same sample to observe the vortex distribution. In the measurement of $J_{c}$, the effect of U-doping was only visible in high magnetic fields. Therefore, it may be difficult to observe the effect in the observation of vortex, which is in the low magnetic field.

\section{CONCLUSION}

We observed the vortex distribution in U-doped and undoped Bi-2223 Ag-sheathed tape applying the high-resolution Bitter method.

1) Effect of the uranium doping on the vortex distribution:

We observed the vortex distribution in $\mathrm{Bi}-2223$ tapes without (sample A) and with uranium doping (sample C, 1.5 wt.\% doping) and irradiated with thermal neu- tron at a fluence of $3 \times 10^{19} \mathrm{~m}^{-2}$. At $25 \mathrm{~K}$, the vortices did not move during Ni deposition (30 seconds), and the vortex distribution for both samples were almost the same. Above $50 \mathrm{~K}$, the vortex moved due to increased thermal energy. The amount of movement was larger for the undoped sample. In both samples no vortex pattern was observed above $75 \mathrm{~K}$.

2) Influence of various thermal neutron fluences on the vortex distribution of 1.5 at.\% U-doped Bi-2223 tapes.

The neutron fluences were $0.75 \times 10^{19} \mathrm{~m}^{-2}$ (sample B) and $3 \times 10^{19} \mathrm{~m}^{-2}$ (sample C). At $25 \mathrm{~K}$, the vortices were trapped by the pinning centers, and did not move in both samples. The vortex pattern of the sample B formed a triangular lattice. Above $50 \mathrm{~K}$, the vortices in both samples moved, and the movement in sample B was slightly larger than in sample $\mathrm{C}$. We did not observe a vortex pattern above $75 \mathrm{~K}$ in both samples.

\section{ACKNOWLEDGMENT}

The authors wish to thank Mr. K. Aizawa for setting up the high-resolution Bitter system and Dr. A. Oota for offering the Bi-2223 silver sheath tapes.

\section{REFERENCES}

[1] H. Sasakura, O. Miura, and D. Ito, "Pb substitution effects on the critical current density in $\mathrm{MgO}$ doped Bi2212 crystals," IEEE Trans. on Applied Superconductivity, vol. 11, pp. 3912-3915, March 2001.

[2] H. Fujii, H. Kumakura, and K. Togano, "Effect of Pb-, La, and Ti-substitutions on the microstructure and superconducting properties of Bi-2212/Ag composite tapes," IEEE Trans. on Applied Superconductivity, vol. 11, pp. 3050-3053, March 2001.

[3] H. Maeda, T. Inaba, M. Sato, P. X. Zhang, and W. P. Chen, "Critical current density and a.c. losses of Ag-sheathed Bi2223 tapes with Sr-V-O barriers," in 13th International Symposium on Superconductivity, (ISS'00) Physica C, vol. 357-360, September 2001, pp. 1230-1233.

[4] M. R. Cimberle, C. Ferdeghini, G. Grasso, P. Gausconi, and A. Malagoli, "Texture development diving the Bi (2223)/Ag multifilamentary tape," IEEE Trans. on Applied Superconductivity, vol. 11, pp. 2987-2990, March 2001.

[5] K. Ohata, J. Sato, M. Okada, K. Tanaka, H. Kitaguchi, H. Kumakura, K. Togano, T. Kiyoshi, and H. Wada, "Effect of filament size on superconducting properties of the Bi-2212 ROSAT wire," in 13th International Symposium on Superconductivity, (ISS'00) Physica C, vol. 357-360, September 2001, pp. 1107-1110.

[6] Y. B. Huang, F. Marti, G. Witz, R. Passerini, R. Flukiger, and G. Grasso, "Enhancing the engineering $J_{c}$ of Bi-2223 multi filamentaly tapes by two-axial rolling and periodic pressing," IEEE Trans. on Applied Superconductivity, vol. 11, pp. 2722-2725, March 2001.

[7] D. G. Marinaro, S. X. Dou, J. Horvat, J. Boldeman, R. Weinstein, and R. Sawh, "Effect of uranium doping and thermal neutron irradiation on the flux-pinning of Silver-Clad Bi-Sr-Ca-Cu-O tapes," IEEE Trans. on Applied Superconductivity, vol. 11, pp. 3896-3899, March 2001.

[8] D. A. Milliken, J. H. Ahn, and S. X. Dou, "Microstructural variations with uranium compound doping of Bi-Sr-Ca-Cu-O/Ag superconducting tapes," IEEE Trans. on Applied Superconductivity, vol. 11, pp. 3964-3967, March 2001.

[9] A. Oota and K. Kawano, "Influence of rolling pressure on microstructures and current path evolution in Ag-sheathed Bi-2223 monocore tapes," IEEE Trans. on Applied Superconductivity, vol. 9, pp. 2529-2532, June 1999.

[10] S. Ohshima, T. Koseki, and A. Kamimura, "Observation of vortex movements in a Bi2212 single crystal under application of a transport current by means of the bitter pattern technique," Jpn. J. Appl. Phys., vol. 37, pp. L 375-378, April 1998.

[11] S. Ohshima, T. Kawai, S. Kanno, and H. Yamada, "Observation of vortex patterns and movement in Y-123 and Bi-2212 sample by means of the bitter technique," Physica C, 2001, to be published. 